\title{
EVIDENCE FOR NEUTRINOLESS DOUBLE BETA DECAY
}

\author{
H.V. KLAPDOR-KLEINGROTHAUS ${ }^{1,3}$, \\ A. DIETZ ${ }^{1}$, H.L. HARNEY ${ }^{1}$, I.V. KRIVOSHEINA ${ }^{1,2}$ \\ ${ }^{1}$ Max-Planck-Institut für Kernphysik, Postfach 1039 80, D-69029 Heidelberg, \\ Germany \\ ${ }^{2}$ Radiophysical-Research Institute, Nishnii-Novgorod, Russia \\ ${ }^{3}$ Spokesman of the GENIUS and HEIDELBERG-MOSCOW Collaborations, \\ e-mail:klapdor@gustav.mpi-hd.mpg.de, \\ home page: http://www.mpi-hd.mpg.de/non_acc
}

\begin{abstract}
The data of the HEIDELBERG-MOSCOW double beta decay experiment for the measuring period August 1990 - May 2000 (54.9813 kg y or 723.44 molyears), published recently, are analyzed using the potential of the Bayesian method for low counting rates. First evidence for neutrinoless double beta decay is observed giving first evidence for lepton number violation. The evidence for this decay mode is $97 \%(2.2 \sigma)$ with the Bayesian method, and $99.8 \%$ c.l. $(3.1 \sigma)$ with the method recommended by the Particle Data Group. The half-life of the process is found with the Bayesian method to be $\mathrm{T}_{1 / 2}^{0 \nu}=(0.8-18.3) \times 10^{25} \mathrm{y}(95 \%$ c.l. $)$ with a best value of $1.5 \times 10^{25} \mathrm{y}$. The deducedvalue of the effective neutrino mass is, with the nuclear matrix elements from $\mathrm{Q},\langle m\rangle=(0.11-0.56) \mathrm{eV}(95 \%$ c.l.), with a best value of $0.39 \mathrm{eV}$. Uncertainties in the nuclear matrix elements may widen the range given for the effective neutrino mass by at most a factor 2. Our observation which at the same time means evidence that the neutrino is a Majorana particle, will be of fundamental importance for neutrino physics. PACS. 14.69.Pq Neutrino mass and mixing - 23.40.Bw Weak-interaction and lepton (including neutrino) aspects - 23.40.-s Beta decay; double beta decay; electron and muon capture.
\end{abstract}

The neutrino oscillation interpretation of the atmospheric and solar neutrino data, deliver a strong indication for a non-vanishing neutrino mass. While such kind of experiments yields information on the difference of squared neutrino mass eigenvalues and on mixing angles, the absolute scale of the neutrino mass is still unknown. Information from double beta decay experiments is indispensable to solve these questions 1110 . Another important problem is that of the fundamental character of the neutrino, whether it is a Dirac or a Majorana particle 2. B. Neutrinoless double beta decay could answer also this question. Perhaps the main question, which can be investigated by double beta decay with high sensitivity, is that of lepton number conservation or non-conservation.

Double beta decay, the rarest known nuclear decay process, can occur in different modes:

KK-Evid-InJ'ModLettA: submitted to World Scientific on October 23, $2018 \mathbf{1}$ 


$$
\begin{array}{ll}
2 \nu \beta \beta-\text { decay : } & A(Z, N) \rightarrow A(Z+2, N-2)+2 e^{-}+2 \bar{\nu}_{e} \\
0 \nu \beta \beta-\text { decay : } & A(Z, N) \rightarrow A(Z+2, N-2)+2 e^{-} \\
0 \nu(2) \chi \beta \beta-\text { decay }: & A(Z, N) \rightarrow A(Z+2, N-2)+2 e^{-}+(2) \chi
\end{array}
$$

While the two-neutrino mode (1) is allowed by the Standard Model of particle physics, the neutrinoless mode $(0 \nu \beta \beta)$ (2) requires violation of lepton number $(\Delta \mathrm{L}=2)$. This mode is possible only, if the neutrino is $\mathrm{p}^{\mathrm{a}}$ Majorana marticle, i.e. the neutrino is its own nntiparticle (E. Majorana Racah

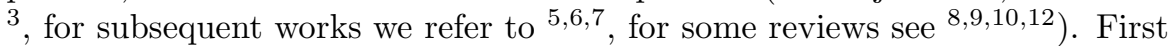
calculations ff $0 \nu \beta \beta$ decay based on the Majorana theory have been done by W.H. Furry $\mathrm{G}$.

Neutrinoless double beta decay can not only probe a Majorana neutrino mass, but various new physics scenarios beyond the Standard Model, such as R-parity violating supersymmetric models, R-parity conserving SUSY models, leptoquarks, violation of Lorentz-invariance, and compositeness (for a review see $10.4,44)$. Any theory containing lepton number violating interactions can in principle lead to this process allowing to obtain information on the specific underlying theory. The experimental signature of the neutrinoless mode is a peak at the Q-value of the decay.

The HEIDELBERG-MOSCOW double beta decay experiment in the Gran Sasso Underground Laboratory searches for double beta decay of ${ }^{76} \mathrm{Ge} \longrightarrow{ }^{76} \mathrm{Se}+2 e^{-}+(2 \nu)$ since 1990. It is the most sensitive double beta experiment since almost eight years now. The experiment operates five enriched (to $86 \%$ ) high-purity ${ }^{76} \mathrm{Ge}$ detectors, with a total mass of $11.5 \mathrm{~kg}$, the active mass of $10.96 \mathrm{~kg}$ being equivalent to a source strength of $125.5 \mathrm{~mol}$ ${ }^{76} \mathrm{Ge}$ nuclei. This is the largest source strength in use.

The high energy resolution of the Ge detectors assures that there is no background for a $0 \nu \beta \beta$ line from the two-neutrino double beta decay in this experiment.

The unique feature of neutrinoless double beta decay is that a measured half-life allows to deduce information on the effective Majorana neutrino mass $\langle m\rangle$, which is a superposition of neutrino mass eigenstates. The half-life is given, when ignoring contributions from right-handed weak currents, by 8 .

$$
\begin{gathered}
{\left[T_{1 / 2}^{0 \nu}\left(0_{i}^{+} \rightarrow 0_{f}^{+}\right)\right]^{-1}=C_{m m} \frac{\langle m\rangle^{2}}{m_{e}^{2}}} \\
\langle m\rangle=\left|m_{e e}^{(1)}\right|+e^{i \phi_{2}}\left|m_{e e}^{(2)}\right|+e^{i \phi_{3}}\left|m_{e e}^{(3)}\right|,
\end{gathered}
$$

Here $\left|m_{e e}^{(i)}\right| \exp \left(i \phi_{i}\right)(i=1,2,3)$ are the contributions to $\langle m\rangle$ from individual mass eigenstates, with $\phi_{i}$ denoting relative Majorana phases connected

KK-Evid-InJ'ModLettA: submitted to World Scientific on October 23, $2018 \mathbf{2}$ 
with $\mathrm{CP}$ violation and, $C_{m m}$ denotes a nuclear matrix element, which can be calculated.

The effective mass is closely related to the parameters of neutrino oscillation experiments. The importance of $\langle m\rangle$ for solving the problem of the structure of the neutrino mixing matrix and in particular to fix the absolute scale of the neutrino mass spectrum which cannot be fixed by $\nu$ - oscillation experiments alone, has been discussed in detail in e.g. 11 .

In this paper, we present a new, refined analysis of the data obtained in the HEIDELBERG-MOSCOW experiment during the period August 1990 May 2000 15. The analysis concentrates on the neutrinoless decay mode which is the one relevant for particle physics (see, e.g. 11.10). A description of the HEIDELBERG-MOSCOW experiment has been given recently in 16 , and in 15.

Fig. 11 shows the combined spectrum of the five enriched detectors obtained over the period August 1990 - May 2000, with a statistical significance of $54.981 \mathrm{~kg}$ y (723.44 molyears), around the Q-value of double beta decay. The latter has been determined to be $\mathrm{Q}_{\beta \beta}=2039.006(50) \mathrm{keV}$ in a recent precision experiment 47 . Fig. 2 2 shows the spectrum obtained with detectors Nr. 1,2,3,5 over the period August 1990 - May 2000, with a significance of $46.502 \mathrm{~kg}$ y. Fig. 3 shows the spectrum of single site events (SSE) obtained for detectors 2,3,5 in the period November 1995 - May 2000, under the restriction that the signal simultanously fulfills the criteria of all three methods of pulse shape analysis we have recently developed 18,29 and with which we operate all detectors except detector 1 (significance $28.053 \mathrm{~kg}$ y). Double beta events are single site events confined to a few $\mathrm{mm}$ region in the detector, corresponding to the track length of the emitted electrons, in contrast to e.g. multiple scattering $\gamma$ - events. From simulation we expect that about $5 \%$ of the double beta single site events should be seen also as MSE. This is caused by bremsstrahlung of the emitted electrons 20 .

All the spectra are obtained after rejecting coincidence events between different Ge detectors and events coincident with activation of the muon shield. The spectra which are taken in bins of $0.36 \mathrm{keV}$ are shown in Figs. 1, 2, 3 in $1 \mathrm{keV}$ bins, which explains the broken number in the ordinate. We do the analysis of the measured spectra with (Fig. 1) and without the data of detector 4 (Figs. 2, 3) since the latter does not have a muon shield and has the weakest energy resolution. We ignore for each detector the first 200 days of operation, corresponding to about three half-lives of ${ }^{56} \mathrm{Co}\left(T_{1 / 2}=77.27\right.$ days $)$, to allow for some decay of short-lived radioactive impurities.

The background rate in the energy range $2000-2080 \mathrm{keV}$ is found to be $(0.17 \pm 0.01)$ events/ kg y keV (without pulse shape analysis) considering

KK-Evid-InJ'ModLettA: submitted to World Scientific on October 23, 20183 


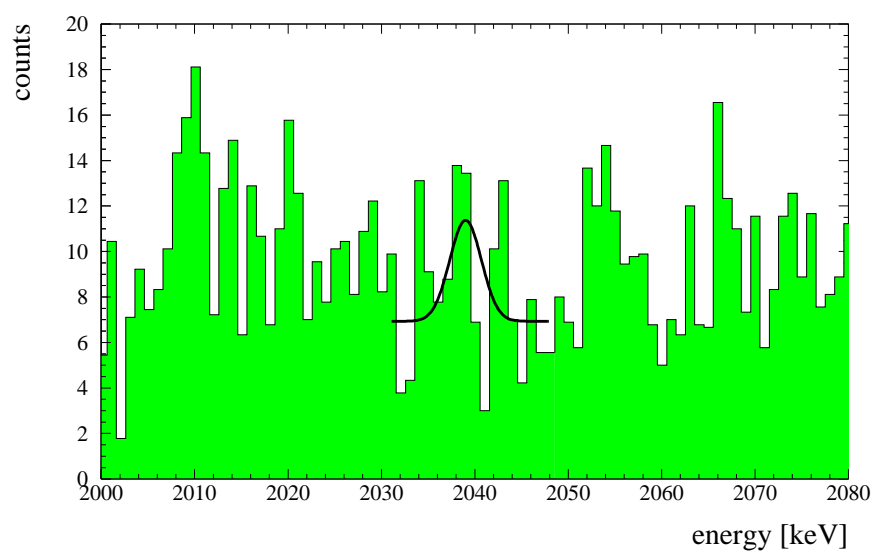

Figure 1. Sum spectrum of the ${ }^{76} \mathrm{Ge}$ detectors Nr. 1,2,3,4,5 over the period August 1990 to May $2000,(54.981 \mathrm{~kg} y)$ in the energy interval $2000-2080 \mathrm{keV}$, around the $\mathrm{Q}_{\beta \beta}$ value of double beta decay $\left(\mathrm{Q}_{\beta \beta}=2039.006(50) \mathrm{keV}\right)$. The curve results from Bayesian inference in the way explained in the text. It corresponds to a half-life $\mathrm{T}_{1 / 2}^{0 \nu}=(0.80-35.07) \times 10^{25}$ (95\% c.l.).

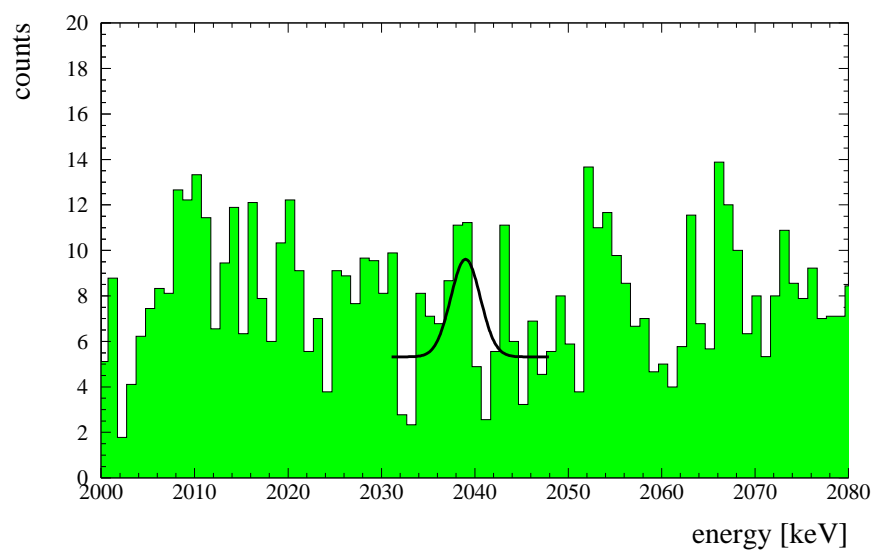

Figure 2. Sum spectrum of the ${ }^{76} \mathrm{Ge}$ detectors Nr. 1,2,3,5 over the period August 1990 to May 2000, $46.502 \mathrm{~kg}$ y. The curve results from Bayesian inference in the way explained in the text. It corresponds to a half-life $\mathrm{T}_{1 / 2}^{0 \nu}=(0.75-18.33) \times 10^{25}$ y $(95 \%$ c.l. $)$.

all data as background. This is the lowest value obtained in such type of 


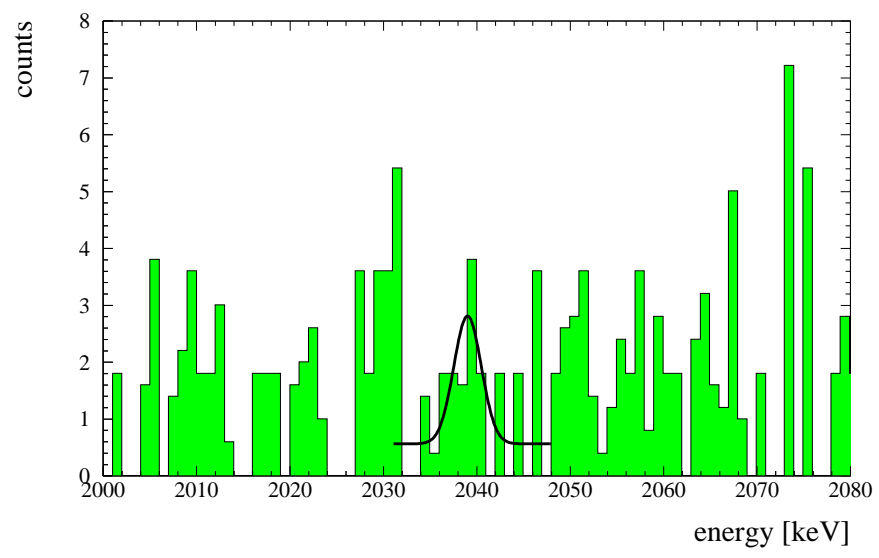

Figure 3. Sum spectrum, measured with the detectors Nr. 2,3,5 operated with pulse shape analysis in the period November 1995 to May $2000(28.053 \mathrm{~kg} \mathrm{y})$, in the region of interest for the $0 \nu \beta \beta$ - decay. Otruts identified as single site events (SSE) by all three pulse shape analysis methods 18,19 have been accepted. The spectrum has been corrected for the efficiency of SSE identification (see text). The curve results from Bayesian inference in the way explained in the text. The signal corresponds to a half-life $\mathrm{T}_{1 / 2}^{0 \nu}=(0.88-22.38) \times 10^{25} \mathrm{y}$ (90\% c.l.).

experiments. The energy resolution at the $\mathrm{Q}_{\beta \beta}$ value in the sum spectra is extrapolated from the strong lines in the spectrum to be $(4.00 \pm 0.39) \mathrm{keV}$ for the spectra with detector 4 , and $(3.74 \pm 0.42) \mathrm{keV}$ (FWHM) for those without detector 4 . The energy calibration of the experiment has an uncertainty of $0.20 \mathrm{keV}$. For more experimental details see 16.15.

We analyse the spectra shown in Figs. 1,2, 2,3 with the following methods:

1. Bayesian method, which is used widely at present (see, e.g. 21). This method is particularly suited for low counting rates, where the data follow a Paisson distribution, that cannot be approximated by a Gaussian (see, also $2223)$.

2. Method recommended by the Particle Data Group 25.

The $\chi^{2}$ - method is not used since it is expected to have some limitation for application at low counting rates, as also the Maximum Likelihood Method. The limitation is due to the Gaussian approximations inherent in these methods.

To be conservative, we first concentrate on the spectra without pulse shape analysis. For the evaluation, we consider the raw data of the detectors. After that we shall turn to the analysis of the spectrum obtained with pulse

KK-Evid-InJ'ModLettA: submitted to World Scientific on October 23, 20185 
shape discrimination.

Figs. 1.5.6 show the result of a Bayesian peak detection procedure. One knows that the lines in the spectrum are Gaussians with a standard deviation $\sigma=1.70$ (1.59) keV corresponding to 4.0 (3.7) keV FWHM. Given the position of a line, we used Bayes theorem to infer the contents of the line and the level of a constant background. We first describe the procedure summarily and then give some mathematical details.

Bayesian inference yields the joint probability distribution for both parameters. The background level was integrated out. This yielded the distribution of the line contents. If the distribution has its maximum at zero contents, only an upper limit for the contents can be given and the procedure does not suggest the existence of a line. If the distribution has its maximum at non-zero contents, the existence of a line is suggested and one can define the probability $\mathrm{K}$ that there is a line with non-zero contents. It is associated with an error interval containing with probability $\mathrm{K}$ the true value of the contents. The Bayesian probability K agrees with the confidence of classical statistics in the case of a Gaussian likelihood function.

For every energy $\mathrm{E}$ of the spectra of Figs. 1,2,3, we have determined the probability $\mathrm{K}$ that there is a line at $\mathrm{E}$. All the remainder of the spectrum was considered to be background in this search. The result is given on the left-hand sides of Figs. $1,5,6$.

We define the Bayesian procedure in some more detail. It starts from the distribution $p\left(x_{1} \ldots x_{M} \mid \rho, \eta\right)$ of the count rates $x_{1} \ldots x_{M}$ in the bins $1 . . \mathrm{M}$ of the spectrum - given two parameters $\rho, \eta$. The distribution $p$ is the product

$$
p\left(x_{1} \ldots x_{M} \mid \rho, \eta\right)=\prod_{k=1}^{M} \frac{\lambda_{k}^{x_{k}}}{x_{k} !} e^{-\lambda_{k}}
$$

of Poissonians for the individual bins. The expectation value $\lambda_{k}$ is the superposition

$$
\lambda_{k}=\rho\left[\eta f_{1}(k)+(1-\eta) f_{2}(k)\right]
$$

of the form factors $f_{1}$ of the line and $f_{2}$ of the background; i.e. $f_{1}(k)$ is the Gaussian centered at $\mathrm{E}$ with the above-mentioned standard deviation and $\mathrm{f}_{2}(\mathrm{k}) \equiv \mathrm{f}_{2}$ is a constant.

Since

$$
\sum_{k=1}^{M} f_{\nu}(k)=1 \quad \text { for } \quad \nu=1,2
$$

KK-Evid-InJ'ModLettA: submitted to World Scientific on October 23, 20186 

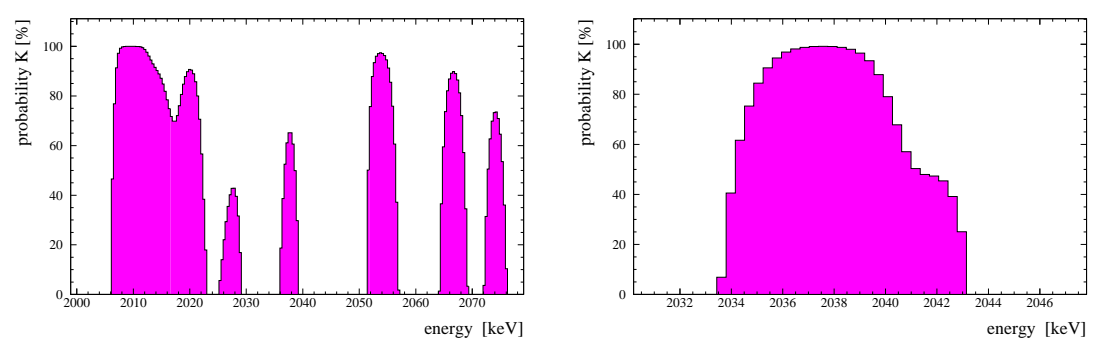

Figure 4. Scan for lines in the full spectrum taken from 1990-2000 with detectors Nr. $1,2,3,4,5$, (Fig. 1), with the Bayesian method. The ordinate is the probability $\mathrm{K}$ that a line exists at energy E. Left: Energy range $2000-2080 \mathrm{keV}$. Right: Energy range of interest around $\mathrm{Q}_{\beta \beta}$.

one has

$$
\sum_{k=1}^{M} \lambda_{k}=\rho .
$$

Hence, $\rho$ parametrizes the total intensity in the spectrum, and $\eta$ is the relative intensity in the Gaussian line.

Prior distributions are given by Jeffreys' rule ( $\$ 5.35$ of 24$)$. The parameter $\rho$ is integrated out and the posterior distribution $\mathrm{P}\left(\eta \mid x_{1} \ldots x_{M}\right)$ of the relative contents $\eta$ of the spectral line is obtained.

The peak detection procedure yields lines at the positions of known weak $\gamma$-lines from the decay of ${ }^{214} \mathrm{Bi}$ at $2010.7,2016.7,2021.8$ and $2052.9 \mathrm{keV}$ t. In addition a line centered at $2039 \mathrm{keV}$ shows up. This is compatible with the Q-value 17,26 of the double beta decay process. We emphasize that at this energy no $\gamma$-line is expected according to the compilations in 27. We do not find indications for the lines from ${ }^{56} \mathrm{Co}$ at $2034.7 \mathrm{keV}$ and $2042 \mathrm{keV}$ discussed earlier 20 (Figs. 呞).

Bayesian peak detection suggests a line at $\mathrm{Q}_{\beta \beta}$ whether or not one includes detector Nr. 4 without muon shield (Figs. 14,5). The line is also suggested in Fig. 6 after removal of multiple site events (MSE), see below.

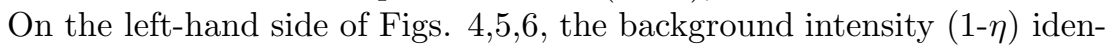
tified by the Bayesian procedure is too high because the procedure averages the background over all the spectrum (including lines) except for the line it is trying to single out. Therefore on the right-hand side of Figs. 4, 5, 6 , the peak detection procedure is carried out within an energy interval that does not contain (according to the left-hand side) lines other than the one at $\mathrm{Q}_{\beta \beta}$. Still the

KK-Evid-InJ'ModLettA: submitted to World Scientific on October 23, 20187 

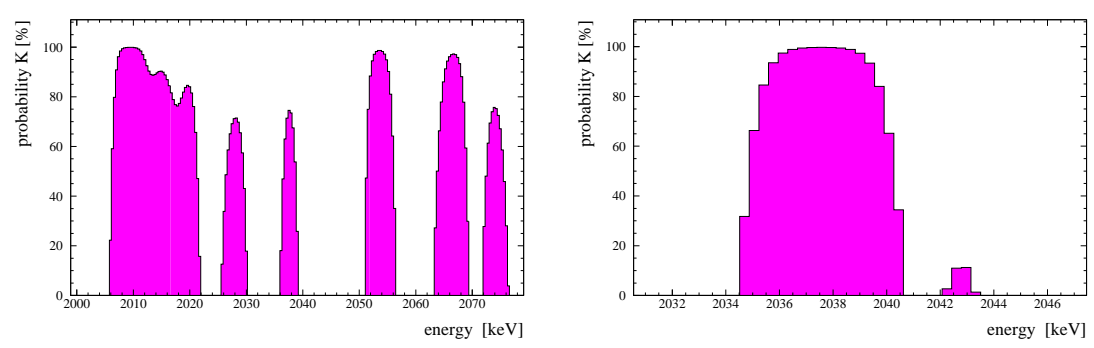

Figure 5. Left: Probability $\mathrm{K}$ that a line exists at a given energy in the range of 2000$2080 \mathrm{keV}$ derived via Bayesian inference from the spectrum shown in Fig. 2. Right: Result of a Bayesian scan for lines as in the left part of this figure, but in the energy range of interest around $\mathrm{Q}_{\beta \beta}$.

interval is broad enough (about \pm 5 standard deviations of the Gaussian line) for a meaningful analysis. We find the probability $\mathrm{K}=96.5 \%$ that there is a line at $2039.0 \mathrm{keV}$ in the spectrum shown in Fig. 1. This is a confidence level of $2.1 \sigma$ in the usual language. For the spectrum shown in Fig. 2, we find a probability for a line at $2039.0 \mathrm{keV}$ of $97.4 \%(2.2 \sigma)$. In this case the number of events is found to be 1.2 to 29.4 with $95 \%$ c.l.. It is 7.3 to 22.6 events with $68.3 \%$ c.l..The most probable number of events (best value) is 14.8 events

We also applied the method recommended by the Particle Data Group 2 . This method (which does not use the information that the line is Gaussian)
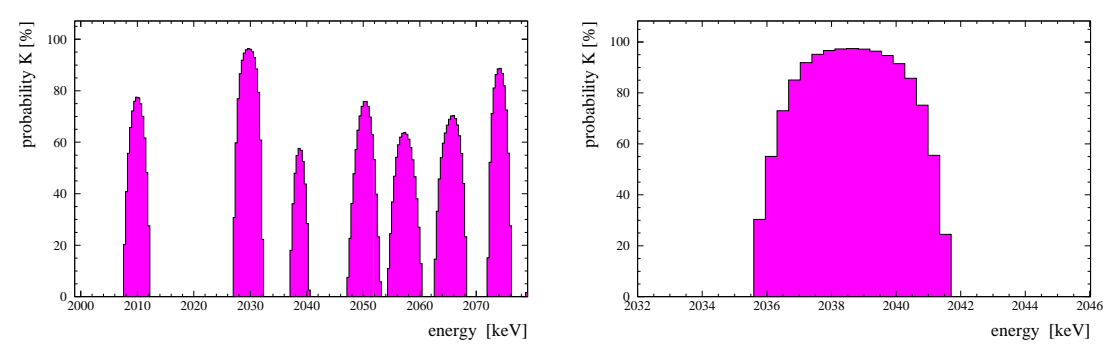

Figure 6. Scan for lines in the single site event spectrum taken from 1995-2000 with detectors Nr. 2,3,5, (Fig. 3), with the Bayesian method (as in Figs. 1, 5). Left: Energy range $2000-2080 \mathrm{keV}$. Right: Energy range of interest around $\mathrm{Q}_{\beta \beta}$.

KK-Evid-InJ'ModLettA: submitted to World Scientific on October 23, 20188 
finds a line at $2039 \mathrm{keV}$ on a confidence level of $3.1 \sigma$ (99.8\% c.l.). Such value is obtained in a wide range of the assumed width of the signal and the energy range of evaluation.

\begin{tabular}{c|c|c|c|c|c}
\hline \hline $\mathrm{N}$ & $\mathrm{N}$ Bin & Energy keV & $\mathrm{N}$ & $\mathrm{N}$ Bin & Energy keV \\
\hline & & & & & \\
1. & 5653 & 2034.66 & 6. & 5666 & 2039.33 \\
2. & 5658 & 2036.46 & 7. & 5669 & 2040.41 \\
3. & 5660 & 2037.18 & 8. & 5674 & 2042.21 \\
4. & 5664 & 2038.61 & 9. & 5680 & 2044.37 \\
5. & 5665 & 2038.97 & & & \\
\hline \hline
\end{tabular}

Table 1. Events classified to be single site events (SSE) by all three methods of PSA, in the range $2034.1-2044.9 \mathrm{keV}\left( \pm 3 \sigma\right.$ range around $\mathrm{Q}_{\beta \beta}$ ) of the spectrum taken with enriched detectors Nr. 2,3,5 in the period November 1995 - May 2000 (28.053 kg y).

From the analysis of the single site events, we find after $28.053 \mathrm{~kg} y$ of measurement 9 SSE events in the region $2034.1-2044.9 \mathrm{keV}( \pm 3 \sigma$ around $\mathrm{Q}_{\beta \beta}$ ) (Table 11). Analysis with the Bayesian method of the single site event spectrum (Fig. 3), as described before, shows again evidence for a line at the energy of $\mathrm{Q}_{\beta \beta}$ (Fig. 6). Analyzing the range of $2032-2046 \mathrm{keV}$, we find the probability of $96.8 \%$ that there is a line at $2039.0 \mathrm{keV}$. We thus see a signal of single site events, as expected for neutrinoless double betadecay, precisely at the $Q_{\beta \beta}$ value obtained in the precision experiment of 47 . The analysis of the line at $2039.0 \mathrm{keV}$ before correction for the efficiency yields 4.6 events (best value) or $(0.3-8.0)$ events within $95 \%$ c.l. $((2.1-6.8)$ events within $68.3 \%$ c.l.). Corrected for the efficiency to identify an SSE signal by successive application of all three PSA methods, which is $0.55 \pm 0.10$, we obtain a $0 \nu \beta \beta$ signal with $92.4 \%$ c.l.. The signal is $(3.6-12.5)$ events with $68.3 \%$ c.l. (best value 8.3 events).

The PDG method gives a signal at $2039.0 \mathrm{keV}$ of $2.8 \sigma(99.4 \%)$. The analysis given in Fig. 6 (left part) shows partly drastic differences in the relative intensities of other identified lines compared to the full spectra, which should reflect their different composition of single site and multiple site events.

We emphasize that we find in all analyses of our spectra a line at the value of $\mathrm{Q}_{\beta \beta}$. Under the assumption that the signal at $\mathrm{Q}_{\beta \beta}$ is not produced by a background line of presently unknown origin, we can translate the observed number of events into half-lives. We give in Table 2 conservatively the values obtained with the Bayesian method and not those obtained with the PDG

KK-Evid-InJ 'ModLettA: submitted to World Scientific on October 23, 20189 
method. Also given in Table 2are the effective neutrino masses $\langle m\rangle$ deduced using the matrix elements of 129.

We derive from the data taken with $46.502 \mathrm{~kg}$ y the half-life $\mathrm{T}_{1 / 2}^{0 \nu}=(0.8-$ $18.3) \times 10^{25}$ y (95\% c.l.). The analysis of the other data sets, shown in Table 2. and in particular of the single site events data, which play an important role in our conclusion, confirm this result.

\begin{tabular}{c|c|c|c|c}
\hline \hline $\begin{array}{c}\text { Significan- } \\
\text { ce }[k g y]\end{array}$ & Detectors & $\mathrm{T}_{1 / 2}^{0 \nu} \mathrm{y}$ & $\langle m\rangle \mathrm{eV}$ & $\begin{array}{c}\text { Conf. } \\
\text { level }\end{array}$ \\
\hline & & & & \\
54.9813 & $1,2,3,4,5$ & $(0.80-35.07) \times 10^{25}$ & $(0.08-0.54)$ & $95 \%$ c.l. \\
54.9813 & $1,2,3,4,5$ & $(1.04-3.46) \times 10^{25}$ & $(0.26-0.47)$ & $68 \%$ c.l. \\
54.9813 & $1,2,3,4,5$ & $1.61 \times 10^{25}$ & 0.38 & Best Value \\
\hline 46.502 & $1,2,3,5$ & $(0.75-18.33) \times 10^{25}$ & $(0.11-0.56)$ & $95 \%$ c.l. \\
46.502 & $1,2,3,5$ & $(0.98-3.05) \times 10^{25}$ & $(0.28-0.49)$ & $68 \%$ c.l. \\
46.502 & $1,2,3,5$ & $1.50 \times 10^{25}$ & 0.39 & Best Value \\
\hline 28.053 & $2,3,5 \mathrm{SSE}$ & $(0.88-22.38) \times 10^{25}$ & $(0.10-0.51)$ & $90 \%$ c.l. \\
28.053 & $2,3,5 \mathrm{SSE}$ & $(1.07-3.69) \times 10^{25}$ & $(0.25-0.47)$ & $68 \%$ c.l. \\
28.053 & $2,3,5 \mathrm{SSE}$ & $1.61 \times 10^{25}$ & 0.38 & Best Value \\
\hline \hline
\end{tabular}

Table 2. Half-life for the neutrinoless decay mode and deduced effective neutrino mass from the HEIDELBERG-MOSCOW experiment.

The result obtained is consistent with the limits given earlier by the HEIDELBERG-MOSCOW experiment 15 , considering that the background had been determined more conservatively there. It is also consistent with all other double beta experiments - which still reach less sensitivity. A second Ge-experiment, which has stopped operation in 1999 after reaching a significance of $9 \mathrm{~kg}$ y yields (if one believes their method of 'visual inspection' in their data analysis) in a conservative analysis a limit of $\mathrm{T}_{1 / 2}^{0 \nu}>0.55 \times 10^{25}$ y $(90 \%$ c.l.). The ${ }^{128} \mathrm{Te}$ geochemical experiment yields $\left\langle m_{\nu}\right\rangle<1.1 \mathrm{eV}$ (68\% c.l.), the ${ }^{130} \mathrm{Te}$ cryogenic experiment yields $\left\langle m_{\nu}\right\rangle<1.8 \mathrm{eV}$ and the $\mathrm{CdWO}_{4}$ experiment $\left\langle m_{\nu}\right\rangle<2.6 \mathrm{eV}$, all derived with the matrix elements of 1 .29 to make the results comparable to the present value (for references see 10 ).

Concluding we obtain, with more than $95 \%$ probability, first evidence for the neutrinoless double beta decay mode.

As a consequence, on the same confidence level, lepton number is not conserved. Further the neutrino is a Majorana particle. We conclude from

KK-Evid-InJ'ModLettA: submitted to World Scientific on October 23, 201810 


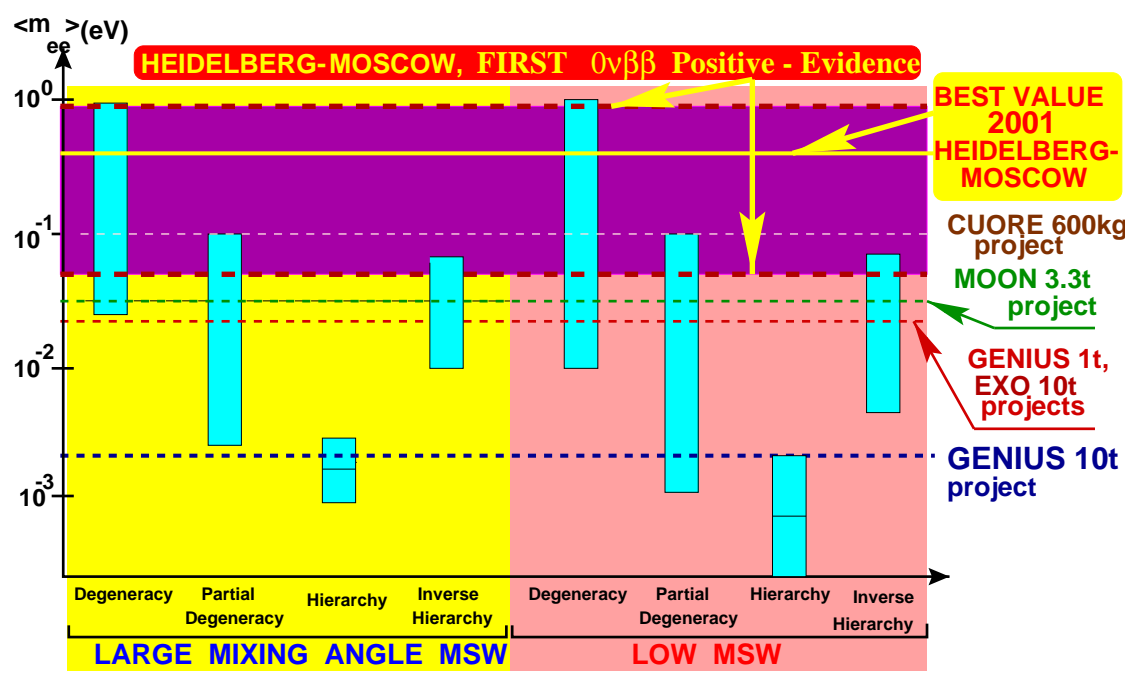

Figure 7. The impact of the evidence obtained for neutrinoless double beta decay in this paper (best value of the effective neutrino mass $\langle m\rangle=0.39 \mathrm{eV}, 95 \%$ confidence range $(0.05$ - 0.84) $\mathrm{eV}$ - allowing already for an uncertainty of the nuclear matrix element of a factor of $\pm 50 \%$ on possible neutrino mass schemes. The bars denote allowed ranges of $\langle m\rangle$ irim different neutrino mass scenarios, still allowed by neutrino oscillation experiments (see 11). Hierarchical models are excluded by the new $0 \nu \beta \beta$ decay result. Also shown are the expected sensitivities for the future potential doubleta experiments CUORE, MOON, EXO and the 1 ton and 10 ton project of GENIUS 31

the various analyses given above the effective mass $\langle m\rangle$ to be $\langle m\rangle=(0.11$ $0.56) \mathrm{eV}$ (95\% c.l.), with best value of $0.39 \mathrm{eV}$. Allowing conservatively for an uncertainty of the nuclear matrix elements of $\pm 50 \%$ (for detailed discussions of the status of nuclear matrix elements we refer to 29.22 .10030$)$ this range may widen to $\langle m\rangle=(0.05-0.84) \mathrm{eV}(95 \%$ c.l. $)$.

In this conclusion, it is assumed that contributions to $0 \nu \beta \beta$ decay from processes other than the exchange of a Majorana neutrino (see, e.g. 28 10 ) are negligible.

With the limit deduced for the effective neutrino mass, the HEIDELBERG-MOSCOW experiment excludes several of the neutrino mass scenarios allowed from present neutrino oscillation experiments (see Fig. 17) allowing mainly only for degenerate and partially degenerate mass scenarios and an inverse hierarchy $3 \nu$ - scenario (the latter being, however, strongly disfavored by a recent analysis of SN1987A. In particular hierarchical mass

KK-Evid-InJ'ModLettA: submitted to World Scientific on October 23, 20181 
schemes are excluded at the present level of accuracy.

Assuming the degenerate scenarios to be realized in nature we fix - according to the formulae derived in 11 - the common mass eigenvalue of the degenerate neutrinos to $\mathrm{m}=(0.05-3.4) \mathrm{eV}$. Part of the upper range is already excluded by tritium experiments, which give a limit of $\mathrm{m}<2.2 \mathrm{eV}$ (95\% c.l.) 34. The full range can only partly (down to $\sim 0.5 \mathrm{eV}$ ) be checked by future tritium decay experiments, but could be checked by some future $\beta \beta$ experiments (see, e.g. 10.11). The deduced best value for the mass is consistent with expectations from experimental $\mu \rightarrow e \gamma$ branching limits in models assuming the generating mechanism for the neutrino mass to be also responsible for the recent indication for as anomalous magnetic moment of the muon 33 . It lies in a range of interest also for Z-burst models recently discussed as explanation for super-high energy cosmic ray events beyond the GKZ-cutoff 32 .

The neutrino mass deduced allows neutrinos to still play an important role as hot dark matter in the Universe.

New approaches and considerably enlarged experiments (as discussed, e.g. in 31) will be required in future to fix the neutrino mass with higher accuracy.

\section{Acknowledgments}

One of the authors - H.V. Klapdor-Kleingrothaus - would like to thank all colleagues who have contributed to the experiment over the last decade of operation. They are particularly grateful to Dr. F. Petry, Dr. B. Maier, Dr. J. Hellmig and Dr. B. Majorovits who have developed the pulse shape discrimination methods and set up the VME electronics applied in the experiment since 1995. They thank Dr. T. Kihm for his highly efficient permanent support in the field of electronics and Dr. G. Heusser for his important contribution in the field of low-level techniques. They thank Mr. H. Strecker for his invaluable technical contributions. They thank Prof. D. Schwalm for his scientific interest and his efficient support of this project and would like to thank the director of the Gran Sasso Underground Laboratory, Prof. E. Bettini and also the former directors of Gran Sasso Profs. P. Monacelli and E. Bellotti, for invaluable support. Our thanks extend also to the technical staff of the Max-Planck Institut für Kernphysik and of the Gran Sasso Underground Laboratory. We thank Perkin Elmer (former ORTEC) Company, and in particular Dr. M. Martini, and Dr. R. Collatz for the fruitful cooperation. We are grateful to Dr. G. Sawitzki and Prof. W. Beiglböck from the Institute for Applied Mathematics of the University of Heidelberg for encouraging

discussions. We acknowledge the invaluable support from BMBF and DFG of

KK-Evid-InJ'ModLettA: submitted to World Scientific on October 23, 201812 
this project. We are grateful to the former State Committee of Atomic Energy of the USSR for providing the enriched material used in this experiment.

\section{References}

1. A. Staudt, K. Muto and H.V. Klapdor-Kleingrothaus, Europh. Lett. 13 (1990) 31.

2. E. Majorana, Nuovo Cimento 14 (1937) 171 - 184.

3. G. Racah, Nuovo Cimento 14 (1937) 322 - 328.

4. W.H. Furry, Phys. Rev. 56 (1939) 1184 - 1193.

5. J. A. Mclennan, Jr. Phys. Rev. 106 (1957) 821,

6. K. M. Case, Phys. Rev. 107 (1957) 307,

7. D. V. Ahluwalia, Int. J. Mod. Phys. A 11 (1996) 1855.

8. M. Doi, T. Kotani and E. Takasugi, Prog. of Theor. Phys. Suppl. 83 (1985) $1-175$.

9. K. Muto and H.V. Klapdor, in "Neutrinos", Graduate Texts in Contemporary Physics", ed. H.V. Klapdor, Berlin, Germany: Springer (1988) 183.

10. H.V. Klapdor-Kleingrothaus, "60 Years of Double Beta Decay - From Nuclear Physics to Beyond the Standard Model", World Scientific, Singapore (2001) $1281 \mathrm{pp}$.

11. H.V. Klapdor-Kleingrothaus, H. Päs and A.Yu. Smirnov, Phys. Rev. D 63 (2001) 073005, and hep-ph/0103076.

12. P.Vogel in "Current Aspects of Neutrino Physics", ed. D.O. Caldwell, Berlin, Heidelberg, Germany: Springer (2001) 177 - 198.

13. H.V. Klapdor-Kleingrothaus, in Proc. of Int. Symposium on Lepton and Baryon Number Violation, Trento, Italy, 20-25 April, 1998, ed. H.V. Klapdor-Kleingrothaus and I.V. Krivosheina, IOP, Bristol, (1999) 251301 and Preprint: hep-ex/9901021

14. H.V. Klapdor-Kleingrothaus, Springer Tracts in Modern Physics, 163 (2000) 69-104, Springer-Verlag, Heidelberg, Germany (2000).

15. H.V. Klapdor-Kleingrothaus et al.,(HEIDELBERG-MOSCOW Collaboration), Eur. Phys. J. A 12 (2001) 147 and hep-ph/0103062.

16. M. Günther et al. (HEIDELBERG-MOSCOW Collaboration), Phys. Rev. D 55 (1997) 54.

17. G. Douysset et al., Phys. Rev. Lett. 86 (2001) 4259 - 4262.

18. J. Hellmig and H.V. Klapdor-Kleingrothaus, Nucl. Instrum. Meth. A 455 (2000) 638 - 644 and J. Hellmig, F. Petry and H.V. KlapdorKleingrothaus, Patent DE19721323A.

19. B. Majorovits and H.V. Klapdor-Kleingrothaus. Eur. Phys. J. A 6

KK-Evid-InJ'ModLettA: submitted to World Scientific on October 23, 201813 
(1999) (1999) 463.

20. B. Maier, Dissertation, November 1995, MPI-Heidelberg; F. Petry, Dissertation, November 1995, MPI-Heidelberg; J. Hellmig, Dissertation, November 1996, MPI-Heidelberg; B. Majorovits, Dissertation, December 2000, MPI-Heidelberg; A. Dietz, Dipl. Thesis, Univ. Heidelberg, 2000 (unpublished), and Dissertation, in preparation.

21. G. D'Agostini, hep-ex/0002055, W. von der Linden and V. Dose, Phys. Rev. E 596527 (1999), and F.H. Fröhner, JEFF Report 18 NEA OECD (2000) and Nucl. Sci. a. Engineering 126 (1997) 1.

22. H.L. Harney, Preprint: physics/0103030.

23. H.L. Harney, "Bayesian Inference. Data Evaluation and Decisions", Monograph in preparation, Springer Verlag, Heidelberg 2002.

24. A. O'Hagan, "Bayesian Inference", Vol. 2B of Kendall's Advanced Theory of Statistics, Arnold, London (1994).

25. D.E Groom et al., Particle Data Group, Eur. Phys. J. C 15 (2000) 1.

26. J.G. Hykawy et al., Phys. Rev. Lett. 67 (1991) 1708.

27. R.B. Firestone and V.S. Shirley, Table of Isotopes, Eighth Edition, John Wiley and Sons, Incorp., N.Y. (1998).

28. R.N. Mohapatra and P.B. Pal, "Massive Neutrinos in Physics and Astrophysics", Singapore, Singapore: World Scientific, World Scientific lecture notes in physics, 41 (1991) 318 pp.

29. T. Tomoda, Rept. Prog. Phys. 54 (1991) 53 - 126.

30. A. Faessler and F. Simkovic, J. Phys. G 24 (1998) 2139 - 2178 and Preprint hep-ph/9901215 (1999) 1 - 32.

31. H.V. Klapdor-Kleingrothaus, hep-ph/0103074 and in Proc. of 2nd Workshop on Neutrino Oscillations and Their Origin (NOON 2000), Tokyo, Japan, 6-18 Dec. 2000, ed: Y. Suzuki et al., World Scientific (2001), Nucl. Phys. Proc. Suppl. 100 (2001) 309 - 313 (hep-ph/0102276), Part. Nucl. Lett. 104 (2001) 20 - 39 (hep-ph/0102319).

32. T.J. Weiler, in Proc. Beyond the Desert 1999, Accelerator, NonAccelerator and Space Approaches, Ringberg Castle, Tegernsee, Germany, 6-12 Juni 1999, edited by H.V. Klapdor-Kleingrothaus and I.V. Krivosheina, IOP Bristol, (2000) 1085 - 1106; H. Päs and T.J. Weiler, Phys. Rev. D 63 (2001) 113015 and hep-ph/0101091.

33. E. Ma and M. Raidal, Phys. Rev. Lett. 87 (2001) 011802; Erratum-ibid. 87 (2001) 159901 and hep-ph/0102255.

34. C. Weinheimer, in Proc. of 3rd International Conference on Dark Matter in Astro and Particle Physics (Dark 2000), Heidelberg, Germany, 10-16 July, 2000, ed. H.V. Klapdor-Kleingrothaus, Springer, Heidelberg (2001) $513-519$.

KK-Evid-InJ'ModLettA: submitted to World Scientific on October 23, 201814 